\title{
Die Zukunft des Arbeitsvertrags in Frankreich - Zwischen Normenzersplitterung und neuem Beschäftigungsstatus
}

Ein Blick in andere europäische Länder zeigt, dass die Aufweichung jahrzehntelang geltender Standards im Arbeitsrecht nicht nur in Deutschland zu beobachten ist. Auch in Frankreich gibt es diese Entwicklung: bereits seit längerer Zeit und in teilweise kaum merklichen Schritten. Mit der Einführung einer neuen Arbeitsvertragsform im letzten Jahr erlebte sie einen vorläufigen Höhepunkt. Eine vergleichbar weitreichende Änderung hat der deutsche Gesetzgeber bisher noch gescheut. Zugleich gibt es in Frankreich bereits eine fortgeschrittene Diskussion über Möglichkeiten zur sozialen Absicherung von Arbeitnehmern, die nicht an den klassischen Arbeitsvertrag gekoppelt ist, sondern auf den gesamten Lebensverlauf abstellt. ${ }^{1}$

\section{Vom unbefristeten Arbeitsvertrag zum Normalarbeitsverhältnis}

Im Frühjahr 2006 wurde ein neuer Arbeitsvertrag für junge Erwachsene von der Villepin-Regierung beschlossen und nach massiven gesellschaftlichen Protesten wieder zurückgenommen (Einzelheiten hierzu: Übersicht 1). Seine Ausrichtung folgte dem - kontrovers diskutierten - Ziel einer Lockerung des Arbeitsrechts. Zwar gilt der unbefristete Arbeitsvertrag mit seinen zahlreichen Absicherungen für Arbeitnehmer immer noch für die Mehrheit der Beschäftigten. Nichtsdestotrotz erleben wir seit 30 Jahren eine Zersplitterung der Beschäftigungsverhältnisse, die den Lohnarbeiterstatus ernsthaft erschüttert. Wie lässt sich diese Entwicklung analysieren? Und vor allem, welche Vorschläge liegen auf dem Tisch, um den Arbeitsvertrag neu zu gestalten und die beruflichen Laufbahnen abzusichern?

Bevor der unbefristete Arbeitsvertrag (CDI, Contrat à durée indéterminé) zum Standard für stabile Beschäftigung wurde, musste er einige Hürden passieren. Seine aktuelle rechtliche Form ist das Ergebnis einer langen Geschichte sozialer Auseinandersetzungen. Im 19. Jahrhundert band der Arbeitsvertrag den Arbeiter nur für befristete Zeit und schützte ihn so vor Sklaverei und Knechtschaft. ${ }^{2}$ Die Forderung der Arbeitnehmer nach höherer Stabilität kam erst am Ende des 19. Jahrhunderts auf. Ihren ersten Niederschlag fand sie $1890 \mathrm{mit}$ der Aufnahme eines unbefristeten Dienstvertrags in den Code Napoléon. Trotzdem stand es beiden Parteien völlig frei, den Vertrag jederzeit einseitig wieder aufzulösen, sodass - Ironie der Geschichte - dieser Vorfahre des CDI viel flexibler erscheint als der damals normale Vertrag, der die beiden Parteien für die Dauer der zu leistenden Arbeit bindet.

Trotz der Zunahme der Lohnarbeit war die Mehrheit der französischen Erwerbsbevölkerung bis 1930 im Handwerk und der Landwirtschaft tätig. Zu Beginn des 20. Jahrhunderts begann man jedoch, die

\footnotetext{
Übersicht 1: Die arbeitsmarktpolitischen Ereignisse in Frankreich

August 2005: „Notplan für Beschäftigung" (Plan d'urgence pour l'emploi)

mit Einführung des Neueinstellungsvertrags (CNE, Contrat nouvelles embauches):

unbefristet, für Betriebe mit weniger als 20 Beschäftigten

- jederzeit kündbar in den ersten 2 Jahren (zum Vergleich CDI/CDD: Probezeit max. 2-6 Monate)

- keine Begründungspflicht für eine Kündigung während der Probezeit

- Kündigungsfrist ist einzuhalten

- Abfindungspflicht (8\% des seit Vertragsbeginn insgesamt ausgezahlten Lohns)

12 Monate Frist für Anfechtung der Kündigung

Januar 2006: Einführung des Ersteinstellungsvertrags (CPE, Contrat première embauche)

Ausweitung der Rechtsform des Neueinstellungsvertrags auf Unternehmen mit mehr als

20 Beschäftigten, aber nur für Beschäftigte unter 26 Jahren,

massive Protestbewegung verhindert die Anwendung des Ersteinstellungsvertrags (Le Friant 2006)

Quelle: Zusammenstellung der Autorin

WSI MITTEILUNGEN
}

durch rechtliche Unterordnung unter einen Arbeitgeber definierte Lohnarbeit klar von der Arbeit der Selbstständigen abzugrenzen. Aus dem kollektiven Willen, den mit der Lohnarbeit verbundenen Risiken $\mathrm{zu}$ begegnen, entstanden gesetzliche und tarifvertragliche Regelungen. Es galt, den aus der Verrichtung körperlicher Arbeit, aus Alter, Krankheit, Schwangerschaft, Arbeitslosigkeit und auch aus der ökonomischen Ungewissheit erwachsenen Risiken entgegenzutreten. Die Berücksichtigung dieser Risiken in dem 1910 gegründeten Code du travail, seit 1919 in den Tarifverträgen und schließlich von 1945 an im System der sozialen Sicherung begründete den modernen Begriff des Beschäftigungsstatus (Supiot 1994).

\section{Die Autorin dankt Adelheid Hege, Stefanie Kremer und Ulrich Zachert für wertvolle Kommentare. \\ 2 Die Abschaffung der Zünfte durch das Le Chape- lier-Gesetz von 1791 hat das Beschäftigungsver- hältnis auf einen vom gemeinen Recht geregelten Vertrag zwischen zwei Individuen beschränkt.}

Florence Lefresne, Wirtschaftswissenschaftlerin am Institut des Recherches Economiques et Sociales (IRES). Arbeitsschwerpunkte: Vergleichende Arbeitsmarktpolitik, Arbeitszeitregulierung, Entwicklung der Anstellungsstandards, Weiterbildungspolitik, lebenslanges Lernen. e-mail : Florence.Lefresne@ires-fr.org

Übersetzt aus dem Französischen von Uta Rüenauver e-mail: rueenauver@aol.com 
In den 1950er und 1960er Jahren des 20. Jahrhunderts, einer Periode außerordentlichen Wachstums in der Geschichte des Kapitalismus, wurde die Lohnarbeit zur Matrix der modernen Gesellschaft, wobei den Arbeitnehmerorganisationen eine wesentliche Rolle zukam (Castel 1995). Der unbefristete Vollzeit-Arbeitsvertrag setzte sich allmählich als Standard abhängiger Beschäftigung durch. Seine unbefristete Gültigkeit zielt einerseits auf den Schutz der Arbeitnehmer als Gegenleistung für ihre Unterordnung unter Unternehmensinteressen; andererseits entspricht er dem Interesse der Arbeitgeber an der Stabilisierung der Belegschaft, die für die Planbarkeit der Arbeitsorganisation nötig war. Der unbefristete Vertrag wird mit drei wesentlichen Merkmalen ausgestattet, die eine stabile Beschäftigung garantieren:

- die Einführung der Kündigungsfrist (1958),

- das Recht auf Abfindung im Kündigungsfall (1967),

- die Pflicht, Entlassungen sowohl bei einer individuellen (1973), als auch einer betriebsbedingten Kündigung (1975) durch die Angabe eines ,wirklichen und ernsthaften Grundes" (cause réelle et sérieuse) zu rechtfertigen.

Die Kündigung des Arbeitsverhältnisses wurde damit zu einem klar geregelten rechtlichen Akt. Das Normalarbeitsverhältnis mit einem einzigen Arbeitgeber findet seinen vollkommensten Ausdruck in Großbetrieben, wo tarifvertragliche Entgeltregelungen die Erwerbsbiografie der Arbeitnehmer strukturieren - oft über das gesamte Berufsleben hinweg.

\section{Die Fragmentierung der Normen in den letzten 30 Jahren}

Die Massenarbeitslosigkeit stellt für die Arbeitnehmer eine direkte Bedrohung dar und wird dadurch zur primären Ursache für die Erosion des Beschäftigungsstatus. Aber die Veränderungen betreffen auch die Arbeit selbst. In den Betrieben forcieren die rasante technische Entwicklung, die Instabilität der Märkte und die scharfen Wettbewerbsbedingungen den Flexibilitätsbedarf. Dabei geht es um zwei Formen von
Flexibilität: interne Flexibilität, die auf einen variablen Einsatz der Arbeitnehmer im Betrieb zielt (Reorganisation der Arbeit, Polyvalenz, verstärkte Autonomie...) und externe Flexibilität, die den Bestand des Arbeitsverhältnisses von der Erfüllung bestimmter Aufgaben abhängig macht. $\mathrm{Zu}$ letzterer gehören befristete Beschäftigung (CDD, contrat à durée déterminé), Zulieferverträge, Verträge mit Selbstständigen. Das ökonomische Risiko wird so teilweise oder vollständig auf Dritte übertragen. In manchen Fällen ist die Risikoübertragung total, z. B. bei den Merchandisers im Großhandel, die ihre Tätigkeit selbst gestalten. Ihre Vergütung richtet sich nach der Anzahl der mit ihnen abgeschlossenen Verträge oder nach dem Betriebsergebnis. Hier wird der Arbeitsvertrag wieder zu einem reinen Handelsvertrag, wie dies bei den Tagelöhnern der Fall war, bevor sich die Lohnarbeit durchsetzte.

Doch schon in den 1970er Jahren begannen sich spezielle Beschäftigungsformen zu entwickeln (Zeitarbeit, befristete Beschäftigungsverhältnisse, Teilzeitarbeit). Normativer Bezugspunkt bleibt das unbefristete Arbeitsverhältnis, das zum allgemeingültigen Rechtsstandard geworden war. Das meint, dass das Gesetz zunächst noch vorschreibt, dass befristete und Zeitarbeitsverträge nur für Arbeiten eingesetzt werden dürfen, die von der üblichen Tätigkeit des Betriebs abweichen. Die Rechtsentwicklung weitet jedoch zum einen die Anwendungsmöglichkeiten dieser Beschäftigungsformen aus (vor allem im Rahmen der Arbeitsmarktpolitik), zum anderen werden die rechtlichen Auflagen in der Praxis oft missachtet. So ist befristete Beschäftigung (einschließlich subventionierter Beschäftigung und Lehrlingsverträgen) in privatwirtschaftlichen Betrieben mit mehr als zehn Arbeitnehmern bei Neueinstellungen zum Regelfall geworden: Mit $73 \%$ der Neueingestellten werden Verträge von kurzer Dauer abgeschlossen (DARES 2005).

Mit einem Anteil befristeter Arbeitsverträge (einschließlich subventionierter und Lehrlingsverträge) an der Gesamtbeschäftigung von 13,3 \% liegt Frankreich im Mittelfeld der EU, zwischen Spanien (33,3\%) und Irland (3,7\%) (Eurostat 2006). Internationale Vergleiche sind jedoch nur begrenzt möglich, da die Bedeutung der einzelnen Beschäftigungsformen eng mit der länderspezifischen Gestaltung sozialer Kompromisse und Normen zusammenhängt (Zachert 2004, S. 76). In Großbritan- nien etwa erklärt sich der sehr begrenzte Rückgriff auf befristete Arbeitsverträge $(5,7 \%)$ aus der großen Flexibilität des permanent contract (der das Kündigungsrecht des Arbeitgebers kaum einschränkt).

Die Zeitarbeit ist die zweite Variante des befristeten Arbeitsverhältnisses. Für junge Menschen ist sie mittlerweile ein wichtiger Zugang zum Arbeitsmarkt. Auch hier gibt es strikte Rahmenbedingungen (befristete Vertretung eines Mitarbeiters, zeitweilige Zunahme des Arbeitsanfalls, Saisonarbeit), die jedoch häufig umgangen werden. So verurteilte im Januar 2004 ein Gericht die Firma Renault dazu, etwa 20 Interimsverträge in unbefristete Verträge umzuwandeln. Einige Zeitarbeitsfirmen, die ihr Image als „Sklavenhändler“ loswerden wollen, bemühen sich zunehmend um eine Rolle als externe Personaldienstleister und gehen damit weit über die Vermittlung befristeter Tätigkeit hinaus (Lefèvre et al. 2002).

Die verschiedenen Formen der Teilzeitarbeit passen die Arbeits- an die Produktionszeiten an und bieten dadurch einen weiteren Weg zur Flexibilisierung, vor allem im Dienstleistungssektor. In Frankreich verbreitete sich die Teilzeit zunächst in der ersten Hälfte der 1980er Jahre. Bis Anfang der 1990er Jahre blieb sie jedoch im Vergleich zu anderen EU-Ländern wie Großbritannien und Deutschland ,im Rückstand“. Seitdem nimmt die Teilzeitbeschäftigung im Durchschnitt jährlich um $1 \%$ zu und betraf 2005 17,5 \% der Erwerbstätigen.

Selbstständige Arbeit (2005: $11 \%$ der erwerbstätigen Bevölkerung) kann verschiedene Formen annehmen, von denen manche in vielen Punkten der Lohnarbeit nahe kommen. Der formale Status der Selbstständigkeit verschleiert sehr oft eine tatsächliche Abhängigkeit. Das ist bei vielen kleinen Subunternehmern der Fall, die von einem einzigen Auftraggeber abhängen. Die Diversifizierung der Einstellungsformen und der Beschäftigungsverhältnisse stellt demnach grundlegende Kategorien des Arbeitsrechts infrage - den formalen Gegensatz zwischen Lohnarbeit und selbstständiger Arbeit, den Betrieb als Ort der Arbeitsverrichtung, Beschäftigung als dauerhafte Beziehung. 


\section{3 Vektoren für die Verände-
rung des Beschäftigungs-
verhältnisses}

Bestimmte Beschäftigtengruppen haben ungewollt - bei der Verbreitung der neuen Normen der Lohnarbeit eine Schlüsselrolle gespielt. Junge Erwerbstätige, Frauen und ältere Beschäftigte sind keineswegs nur „periphere" Segmente des Arbeitsmarktes; sie machen zahlenmäßig die Mehrheit der Beschäftigten aus. An ihnen vollziehen sich die Veränderungen innerhalb des Beschäftigungsverhältnisses, die darauf abzielen, das Lohnarbeitsverhältnis insgesamt neu zu ordnen und zugleich die Konturen einer Neusegmentierung des Arbeitsmarktes zu definieren (IRES 2005).

Als Berufseinsteiger sind junge Arbeitnehmer ganz besonders von befristeter Beschäftigung betroffen: In der Privatwirtschaft muss sich mehr als jede(r) Dritte mit einem befristeten Arbeitsvertrag begnügen, noch mehr sind es im nicht privatwirtschaftlichen Bereich. Mit zunehmendem Alter sinkt zwar der Anteil der befristet Beschäftigten, dennoch stellt sich die Frage nach den Langzeitwirkungen dieser neuen, flexibleren Beschäftigungsformen, die häufig zu wiederholter Arbeitslosigkeit führen. Statistiken zeigen, dass der Anteil stabiler Beschäftigungsverhältnisse von einer $\mathrm{Ge}-$ neration zur nächsten abnimmt (Fondeur/ Minni 2005). Durch diesen Generationeneffekt werden junge Berufstätige zum entscheidenden Vektor für den Wandel der Arbeit.

Die Frauen, deren Beschäftigungsrate seit 20 Jahren ständig gewachsen ist und deren durchschnittliches Ausbildungsni- veau mittlerweile das der Männer übersteigt, sind die Hauptbetroffenen der Teilzeitbeschäftigung (2005: $31 \%$ der berufstätigen Frauen im Alter von 15-59 Jahren gegenüber $6 \%$ der Männer gleichen Alters). Umfang und Bedeutung dieser Beschäftigungsform variieren je nach Alter, Qualifikation und Form des Arbeitsvertrags (Letrémy et al. 2004). Teilzeitarbeit betrifft mehrheitlich geringqualifizierte Tätigkeiten mit beschränkten Aufstiegsmöglichkeiten und mit oft zerstückelten Arbeitszeiten und -rhythmen. Die Benachteiligung der Frauen ist hier kumulativ: Sie wirkt sich sowohl auf den Lohn als auch auf das künftige Rentenniveau und die Berufserwartungen aus. Gleichzeitig trägt die Teilzeitarbeit zur Destabilisierung des SMIC (Salaire minimum interprofessionnel de croissance, gesetzlicher Mindestlohn) als Einkommensgarantie bei. Da der SMIC auf einem Stundensatz beruht, reicht er für viele Menschen nicht aus, um der Armut trotz Arbeit zu entgehen. Auch hier sind Frauen die Hauptbetroffenen: 2003 erhielten 3,5 Mio. Beschäftigte einen Lohn unter dem Monats-SMIC, darunter $80 \%$ Frauen.

Die Entwicklung der Erwerbsbevölkerung und die Finanzierungsprobleme der Rentenversicherung haben dazu beigetragen, dass Arbeit im Alter zu einem zentralen gesellschaftlichen Thema geworden ist, dessen sich insbesondere auch die Europäische Kommission bemächtigt. Nachdem ältere Erwerbstätige lange durch Frühverrentungsprogramme vom Arbeitsmarkt verdrängt worden sind, wird ihre Teilnahme heute wieder gefordert - allerdings im Rahmen spezieller Beschäftigungsverhältnisse (Teilzeitarbeit, befristete Beschäftigung). Sie werden zwar seltener befristet eingestellt als ihre jungen Kolleginnen und Kollegen; befristete Beschäftigung ist je-

\begin{tabular}{lccc|}
\hline Tabelle 1: Verteilung der Beschäftigungsformen & $\mathbf{2 0 0 5}-$ in $\%$ - \\
\hline & Frauen & Männer & Gesamt \\
\hline Selbstständige Tätigkeit & 7,5 & 13,8 & 10,9 \\
Unbefristete Vollzeittätigkeit & 55,4 & 71,9 & 64,4 \\
Unbefristete Teilzeittätigkeit & 23,8 & 3,0 & 12,6 \\
"Prekäre Lohnarbeit" & 13,3 & 11,2 & 12,2 \\
$\quad$ darunter : Zeitarbeit & 1,4 & 2,8 & 2,2 \\
Befristete Beschäftigung & 8,7 & 5,3 & 6,9 \\
Praktika und subventionierte Verträge & 2,2 & 1,4 & 1,7 \\
Lehrlinge* & 0,9 & 1,7 & 1,3 \\
\hline Gesamt & 100,0 & 100,0 & 100,0 \\
Anteil der Teilzeit an der Gesamtbeschäftigung & 31,0 & 6,0 & 17,0 \\
\hline * In Frankreich hat "apprentissage" (Lehre/Ausbildung) nicht die gleiche Bedeutung wie in Deutschland; der Begriff ist enger \\
gefasst und umfasst insbesondere niedrig qualifizierte und nicht tarifvertraglich geregelte Ausbildungsverhältnisse. \\
Quelle: INSEE nach Concialdi (2006). & & WS I MITTEILUNGEN
\end{tabular}

doch bei den über 50-Jährigen häufiger als bei 30- bis 49-Jährigen (Jolivet 2003). Im August 2006 wurde ein auf 18 Monate befristeter, einmal verlängerbarer SeniorenVertrag für Arbeitslose über 57 Jahre eingeführt, der diese Entwicklung noch verstärken wird.

Mit über 2 Mio. subventionierten Arbeitsplätzen (emplois aidés) hat die Beschäftigungspolitik entscheidend zur Verharmlosung der befristeten und Teilzeitarbeit beigetragen. Das Experimentieren mit neuen Standards sowie deren Verbreitung wirken sich nicht nur auf die Rechtsform des Beschäftigungsverhältnisses aus. Die Freistellung von Sozialabgaben entlastet den Arbeitgeber in großem Ausmaß von seiner Verantwortung als Mitträger des Sozialversicherungssystems, dessen Finanzierung nun auf die Allgemeinheit übertragen wird. Diese Entlastung ist bei der Einstellung von Arbeitnehmern im Niedriglohnbereich massiv: Sie beläuft sich auf 26 \% für Löhne in der Höhe des SMIC und wirkt degressiv weiter bis zu $1,7 \times$ SMIC. Bei subventionierten Verträgen im nichtprivatwirtschaftlichen Sektor führt die Übernahme fast aller Kosten durch den Staat zur Verzerrung des Arbeitsrechts: Welchen Marktwert hat ein Beschäftigter, der seinen Arbeitgeber praktisch nichts kostet? Ganz allgemein stellt sich die Frage nach dem Sinn einer staatlichen Politik, die die Entwicklung von minderwertigen Beschäftigungsverhältnissen fördert, die den Betroffenen nur provisorische Lösungen anbieten, ohne sie dauerhaft vor Arbeitslosigkeit zu schützen. 2005 erhielten 8,5 Mio. Arbeitnehmer die sogenannte Beschäftigungsprämie (prime à l'emploi): Sie wird vom Staat an Personen gezahlt, die eine neue Arbeit aufnehmen bzw. bereits beschäftigt sind und deren Haushaltseinkommen einen bestimmten Grenzwert ${ }^{3}$ nicht überschreitet. Die Prämie soll vor Armut in der Arbeit schützen, befreit aber gleichzeitig den Arbeitgeber von der Verantwortung, einen existenzsichernden Lohn zu zahlen, den der Stunden-SMIC nicht mehr garantieren kann.
3 Für ein Ehepaar mit zwei Kindern z. B. darf das zu versteuernde Einkommen $32.177 €$ im Jahr nicht überschreiten. Die Prämie kann von den Steuern abgezogen werden, wenn die Person steuerpflich- tig ist; meist handelt es sich jedoch um eine So- zialleistung


Die Ausbreitung atypischer Beschäftigungsformen führt zu einer grundsätzlichen Debatte über die Qualität der Beschäftigung, die auch auf der Agenda der Europäischen Kommission steht. Einerseits erhöhen die atypischen Beschäftigungsformen das Arbeitsangebot im Niedriglohnsektor (unqualifizierte Arbeiter und Angestellte), dessen Zunahme seit Mitte der 1990er Jahre durch verschiedene Untersuchungen belegt ist (Freyssinet 2002; Méda/ Vennat 2004). Die Politik der Entlastung geringfügiger Einkommen von Sozialabgaben (in Höhe von $20 \mathrm{Mrd}$. €) hat diese Entwicklung in hohem Maße gefördert. Andererseits stellt sich die Frage der Rückwirkung dieser besonderen Beschäftigungsformen auf die Arbeitsleistung als Bedingung dauerhaften Wirtschaftswachstums. Tatsächlich stößt hier die Flexibilität des Arbeitsverhältnisses an eine entscheidende Grenze: Die Leistungsbereitschaft der Beschäftigten setzt ein Minimum an Statussicherheit voraus.

\section{Dualisierung des Arbeits- marktes und "Destabilisie- rung der Stabilen"}

In einer zunehmend fragilen Beschäftigungssituation befinden sich in erster Linie Beschäftigte mit kurzer Betriebszugehörigkeit (unter einem Jahr), die erst kurze Zeit Berufstätigen sowie befristet Beschäftigte. Für Letztere ist die Gefahr, den Arbeitsplatz zu verlieren bei sonst gleichbleibenden Bedingungen seit Beginn der 1970er Jahre, um einen Faktor von fast 3,5 gestiegen, während sie bei Erwerbstätigen mit mehr als zehn Jahren Betriebszugehörigkeit stabil bleibt (Behaghel 2003). Der Graben zwischen befristet und nicht befristet Beschäftigten hat sich noch dadurch vertieft, dass die Übergänge zwischen befristeten und Standardarbeitsverhältnissen in letzter Zeit zunehmend fließend geworden sind (Galtier/Gautié 2003). Längerfristiges Verbleiben im „Prekariat" ist keineswegs selten: 8 bis $10 \%$ der Berufstätigen waren in den letzten drei Jahren mindestens einmal arbeitslos oder in einem „prekären Arbeitsverhältnis“ beschäftigt. In Frankreich ist (anders als in Deutschland) das Auslaufen eines befristeten Arbeitsvertrags der Hauptgrund für den Zugang zur Arbeitslosigkeit. In den 1980er Jahren verharrten $20 \%$ der „prekär" Beschäftigten über ein Jahr in dieser Beschäftigungssituation; heute sind es 30 \% (Fougère/Sidhoum 2006). Internationale Vergleiche zeigen, dass in Frankreich die Wahrscheinlichkeit für eine(n) Arbeitslose(n), rasch eine neue Stelle zu finden, geringer ist als im europäischen Durchschnitt (CERC 2005).

Die zunehmende Ungleichheit innerhalb der Arbeitnehmerschaft könnte zu der Vermutung verleiten, dass (vor allem in Großbetrieben) unbefristet Beschäftigte dem gestiegenen Konkurrenzdruck widerstehen können und Beschäftigungsgarantien inne hätten. Tatsächlich aber sind selbst die Insider (qualifizierte Arbeitnehmer mit unbefristeten Vollzeitverträgen) immer mehr dem Druck von Mehrarbeit, Erfolgszwang und Kundenansprüchen ausgesetzt; auch sie sind nicht mehr vor der Drohung des Arbeitsplatzverlusts geschützt, die eine entgrenzte Arbeitsbereitschaft hervorruft (Coutrot 1998). Verschiedene Untersuchungen zeigen, dass verstärkte Autonomie- und Polyvalenzanforderungen mit der Zunahme von Stress und Leiden in der Arbeit einhergehen (Dejours 1998; Paugam 2000). Die normalerweise mit dem unbefristeten Beschäftigungsverhältnis assoziierte Arbeitsplatzsicherheit kann sich als trügerisch erweisen, wie der Anstieg betriebsbedingter und individueller Kündigungen zeigt, deren stigmatisierende Wirkung oft auf die gesamte weitere Erwerbsbiografie der Betroffenen ausstrahlt (Palpacuer et al. 2007). Gleichzeitig werden in den Personalbüros Strategien entwickelt, die darauf abzielen, hoch qualifizierte Mitarbeiter zu halten oder zu gewinnen, indem ihnen maßgeschneiderte Arbeitsverträge und individuell ausgehandelte Gehälter mit beträchtlichen Gewinnbeteiligungen angeboten werden. Für die betroffenen Beschäftigten birgt dieses Modell der Individualisierung des Arbeitsverhältnisses jedoch das Risiko, dass ihre Kompetenzen einzig vom Markt evaluiert werden und allein von ihrem individuellen Verhandlungsgeschick abhängen, ohne dass kollektive Mechanismen der Qualifikationsanerkennung Schutz gewähren (Fondeur/Sauviat 2002).

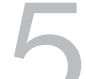 \\ Auf der Suche nach neuen Rechtsgrundlagen}

Die Zersplitterung der Normen geht mit einer starken Destabilisierung des Sozialversicherungssystems einher. Historisch ist die Teilhabe am System der sozialen Sicherheit bei vielen Leistungen an die Art des Beschäftigungsverhältnisses gebunden. Und auch die Arbeitslosenversicherung erreicht heute nur noch $40 \%$ der Arbeitslosen. Dieser Rückgang liegt zu einem großen Teil an einer langen Reihe von Reformen, die sich besonders negativ auf Beschäftigte mit kurzer Beschäftigungsdauer auswirken, deren Arbeitslosengeldbezug gekürzt wird - oder die ganz aus der Arbeitslosenversicherung herausfallen. Viele sehen sich deshalb gezwungen, das soziale Mindesteinkommen (RMI, revenu minimum d'insertion, Mindesteinkommen zur beruflichen Eingliederung) zu beantragen, das im März 2006 an 1,1 Mio. Menschen ausgezahlt wurde. Das Rentensystem und seine jüngsten Reformen konfrontieren die Versicherten mit dem gleichen Problem: „Prekäre", von Arbeitslosigkeit unterbrochene Beschäftigung und Teilzeitarbeit reißen große „Löcher" in das Netz der sozialen Sicherheit.

Mitte der 1990er Jahre schlägt der Boissonnat-Bericht (1995) aus dem Commissariat Général du Plan (Generalkommissariat für Planung) die Schaffung einer neuen Beschäftigungsform vor, den sogenannten Erwerbsvertrag (contrat d'activité). Dieser zielt erstens darauf ab, den Bezugsrahmen des Arbeitsverhältnisses zu erweitern: „Das heutige Arbeitsrecht begrenzt die individuelle Arbeitsbeziehung auf einen Vertrag, der den Arbeitnehmer an einen Arbeitgeber bindet, der in rechtlicher Hinsicht als Vertreter eines einzigen Unternehmens definiert ist. Damit ignoriert es weitgehend die Verbreitung unternehmensübergreifender ökonomischer und sozialer Verantwortung, vor allem im Rahmen von Zuliefer- und Ko-Auftragsbeziehungen " (ebd., S. 283). Tatsächlich bietet diese Erweiterung des Arbeitsvertrags eine Alternative zum System der internen und externen Flexibilität, indem sie eine auf mehrere Unternehmen aufgeteilte Flexibilität befürwortet. Der Vertrag soll es zweitens den Beschäftigten ermöglichen, in ihrer individuellen Erwerbsbiografie Mobilität und Kon- 
Zeitraum erhält der Arbeitsuchende eine Bezahlung in Höhe von 90 \% seines früheren Entgelts.

\subsection{BERUFLICHE SOZIALVERSICHERUNG}

Die Idee einer „beruflichen Sozialversicherung" (sécurité sociale professionnelle) wurde 2002 von der Gewerkschaft CGT (Conféderation générale du travail) entwickelt. Inzwischen wurde sie von vielen Experten, Politikern und sozialen Akteuren aufgegriffen. Hinter dem Begriff verbergen sich jedoch sehr unterschiedliche Vorstellungen im Hinblick auf das Beschäftigungsverhältnis.

Die berufliche Sozialversicherung zielt auf die Schaffung eines neuen Lohnarbeiterstatus als Sockel neuer berufsübergreifender Rechte: Recht auf Weiterbildung, auf Gesundheit, Rente, Arbeit, eine berufliche Karriere, Recht auf soziale Demokratie. Diese Rechte wären an die Person des Arbeitnehmers gebunden, unabhängig davon, $\mathrm{ob}$ dieser für einen oder mehrere Arbeitgeber tätig ist; sie könnten von einem Betrieb auf den anderen übertragen und jedem Arbeitgeber gegenüber geltend gemacht werden. Im Fall einer Umstrukturierung des Unternehmens würden Arbeitsvertrag und Entgeltbedingungen so lange weiter gelten bis der Arbeitnehmer tatsächlich in ein neues, dem vorangegangenen mindestens gleichwertiges Beschäftigungsverhältnis eingegliedert ist. Viele Fragen sind jedoch offen, wie z. B. die Finanzierung und Implementierung der genannten Rechte. Manche schlagen vor, einen neuen Zweig der „beruflichen Sozialversicherung " innerhalb des Sozialversicherungssystems zu schaffen. Andere verweisen auf das Beispiel von auf lokaler Ebene entwickelten Modellen, wie Arbeitgeberzusammenschlüsse oder Wiedereingliederungsgesellschaften, deren Effizienz auf der Dynamik des territorialen sozialen Dialogs beruhen soll.

Die Gewerkschaft CFDT (Confédération française démocratique du travail) hat sich ihrerseits auf ihrem Kongress im Juni 2006 für die Einrichtung kollektiver Garantien ausgesprochen, die den beruflichen Werdegang absichern sollen. Verschiedene Forderungen wurden vorgestellt: So soll über ein System der Anerkennung beruflicher Erfahrung (validation des acquis d'expérience, VAE) und die Ausweitung des individuellen Rechts auf Weiterbildung (droit individuel de formation) die Übertragbarkeit der betrieblich erworbenen Qualifikationen gewährleistet werden; der Anspruch auf die Leistungen der Zusatzkassen im Sozialversicherungssystem soll systematisch weitergelten; für Branchenregelungen soll ein branchenübergreifendes Äquivalenzsystem eingerichtet werden.

Auf das Projekt einer beruflichen Sozialversicherung beruft sich auch der damalige Parteivorsitzende der UMP (Union pour un mouvement populaire) und jetzige Staatspräsident Sarkozy, ${ }^{6}$ der die Flexibilisierung der Beschäftigung als Mittel zur Bekämpfung der Arbeitslosigkeit empfiehlt und im Gegenzug für die Schaffung eines unbefristeten "Gesamtarbeitsvertrags" (contrat de travail unique) anbietet. Die Rechte würden mit der Beschäftigungsdauer wachsen; die Höhe der Abfindungen etwa wäre von der Dauer der Betriebszugehörigkeit abhängig. ${ }^{7}$ Es bestünde ein Recht auf individuelle Wiedereingliederung ins Berufsleben, das effizienter als bisher von den staatlichen Arbeitsagenturen gestaltet würde. Die "sécurité sociale professionnelle" würde ebenfalls die Festsetzung des Arbeitslosengeldes nach individuellen Kriterien erlauben; seine Bezugsdauer würde nach den „objektiven“ individuellen Schwierigkeiten der Wiedereingliederung gestaffelt. Die Mittel kämen aus der Besteuerung betrieblicher Kündigungen, wodurch ein Anreiz für interne Umsetzungen geschaffen wäre. Dieser „Flexicurity“-Ansatz beruft sich auf das berühmte dänische Modell, das in der laufenden Diskussion oft zitiert wird. Die Flexibilität der Beschäftigungsverhältnisse in Dänemark übt eine starke Faszination aus - wobei oft vergessen wird, dass dort die Erwerbssicherheit durch ein hohes Niveau von Ausbildung und sozialer Sicherung ermöglicht wird. So beträgt das Arbeitslosengeld, das vier Jahre lang bezahlt wird, $90 \%$ des vorherigen Einkommens bei einer Obergrenze von $2.000 €$. Umfangreiche Umverteilungskomponenten zur Begrenzung der Ungleichheiten und ein starkes Tarifvertragssystem sind Kernpunkte dieses Modells, die jedoch in der Diskussion leicht ausgeblendet werden.

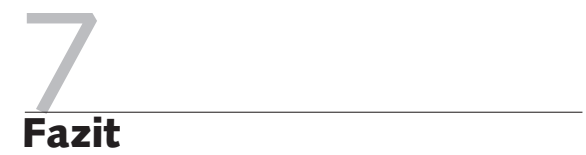

Hinter der von vielen geteilten Diagnose, dass sich der Arbeitsmarkt zunehmend dualisiert, stehen sehr kontroverse Einschätzungen, die sich im Wesentlichen auf zwei Argumentationsstränge konzentrieren.

Ein erster Streitpunkt betrifft das Arbeitsrecht. Inwieweit setzt die erstrebte höhere Fluidität des Arbeitsmarkts die Reform der geltenden Beschäftigungsverhältnisse (befristete/unbefristete Arbeit) und Rechtsnormen (Kündigungsschutz) voraus? Zahlreiche Stimmen plädieren für die Fusion des befristeten und des unbefristeten Arbeitsvertrags, also für die Schaffung einer einheitlichen unbefristeten Vertragsform mit gestaffelten Senioritätsrechten. Dies soll dem Dualisierungseffekt am Arbeitsmarkt entgegenwirken, der von der Existenz dieser beiden Standards ausgeht. Wären damit aber die massiven Ungleichheiten in Bezug auf das Arbeitslosigkeitsrisiko behoben, die vor allem aus unterschiedlichen Qualifikationsniveaus und unterschiedlicher Dauer der Betriebszugehörigkeit erwachsen? Auch eine Lockerung des Kündigungsschutzes wird keineswegs einstimmig befürwortet, wie der jüngste Konflikt um den Ersteinstellungsvertrag (CPE, Contrat première embauche; Übersicht 1) gezeigt hat. Zudem ist fraglich, $\mathrm{ob}$ eine solche Lockerung tatsächlich $\mathrm{zu}$ dem erhofften Beschäftigungszuwachs führt (OECD 2004; Husson 2006).

Der zweite Streitpunkt betrifft die Frage, wie soziale Verantwortung für Beschäftigung wahrgenommen wird. In den meisten hier zitierten Berichten wird empfohlen, Kündigungen zu besteuern, worüber sicherlich weiter nachgedacht werden muss. Die abschreckende Wirkung einer solchen Maßnahme erscheint jedoch zweifelhaft, wenn man an das Beispiel der Einstellung von Behinderten denkt: Die meisten Arbeitgeber ziehen es vor, Bußgelder

\footnotetext{
6 Nicolas Sarkozy: „Retrouvons le plein-emploi grâce à la Sécurité sociale professionnelle" (Zurück zur Vollbeschäftigung dank der Beschäftigungsversicherung), La Tribune, 12. Dezember 2005.

7 In dieselbe Richtung geht der Vorschlag eines „progressiven Vertrags" (contrat progressif) der Fraktion der Union centriste-UDF. Der Vorschlag enthält im Einzelnen: eine sechsmonatige Probezeit, einen Anspruch auf eine Begründung für die Beendigung eines Arbeitsverhältnisses, mit den Berufsjahren wachsende Arbeitnehmerrechte (vor allem Höhe der Abfindung), im Fall der Kündigung Recht auf Validierung der Berufserfahrung (Arbeitszeugnis), auf eine "Kompetenzbilanz" und auf Betreuung durch die ANPE (Arbeitsagentur).
} 
zu bezahlen. Könnten sich die Arbeitgeber solchermaßen „loskaufen“, wären sie zugleich von jeglicher sozialer Verantwortung für Entlassungen befreit. Andere Protagonisten kritisieren solche Vorschläge und fordern im Gegenteil, die Arbeitgeber auf ihre soziale Verantwortung vor, während und nach der Umstrukturierung von Unternehmen zu verpflichten, was Verhandlungen voraussetzt, um die Folgen abzuschätzen, die Mobilität der Arbeitnehmer aktiv vorzubereiten und gegebe- nenfalls auf die Qualität der beruflichen Wiedereingliederungsmaßnahmen einzuwirken. Ganz allgemein stellt sich hier die Frage nach einer neuen kollektiven Bestimmung des Beschäftigungsverhältnisses.

\section{ITERATUR}

Behaghel, L. (2003): Insécurité de l'emploi: le rôle protecteur de l'ancienneté a-t-il baissé ?, Document de travail du CEE 24, Noisy-le-Grand Blanchard, O./Tirole, J. (2003): Protection de l'emploi et procédures de licenciement, Rapport du Conseil d'analyse économique, http://www. cae.gouv.fr

Boissonnat J. (1995): Le travail dans vingt ans, Rapport au Commissariat Général du Plan, Odile Jacob, Paris

Cahuc, P./Kramarz, F. (2004): De la précarité à la mobilité : vers une sécurité sociale professionnelle, Rapport au Ministre de l'Economie, des Finances et de l'Industrie et au Ministre de l'Emploi, du Travail et de la Cohésion sociale, http://www.ladocumentationfrancaise.fr/rapportspublics/054000092/index.shtml

Camdessus, M. (2004): Le sursaut, Vers une nouvelle croissance pour la France, Rapport au Ministre de l'Economie, des Finances et de I'Industrie, http://www.ladocumentationfrancaise.fr/rapports-publics/044000498/ index.shtml

Castel, R. (1995): Les métamorphoses de la question sociale, une chronique du salariat, Fayard, Paris

CERC (2005): La sécurité de l'emploi face aux défis des transformations économiques, Rapport 5, La Documentation française, Paris Concialdi, P. (2006): La France précarisée, un état des lieux, in: Husson (dir.) (Travail précaire, salarié jetable, coll. Sur le Vif, La Découverte, Paris), S. 17-27

Coutrot, T. (1998): L'entreprise néo-libérale, nouvelle utopie capitaliste, coll. Textes à l'appui-Série économique, La Découverte, Paris DARES (2005): Les mouvements de main-d'œuvre au quatrième trimestre 2004, Premières Synthèses-Premières Informations, 32-2

Dejours, C. (1998): Souffrance en France, Seuil, Paris Eurostat (2006): Emploi en Europe 2005, DG Emploi et affaires sociales, Commission Européenne

Fondeur, Y./Sauviat, C. (2002): Normes d'emploi et marché du travail dans les métiers liés aux technologies de l'information, Document d'études, 63, Dares, Paris

Fondeur Y./Minni C. (2005): Les jeunes au cœur des dynamiques du marché du travail, Economie et Statistique, 378-379, S. 85-104 Fougère, D./Sidhoum N. (2006): Les nouvelles inégalités de revenus et I'intégration sociale, Horizons Stratégiques, 2, S. 1-17 Galtier, B./Gautié, J. (2003): Flexibility, stability and the interaction between employment protection and labour market policies in France, in: Auer, P./ Cazes, S. (eds) : Employment stability in an age of flexibility: Evidence from industrialized countries, ILO, Genf Husson, M. (dir.) (2006): Travail précaire, salarié jetable, coll. Sur le Vif, La Découverte, Paris IRES (2005): Les mutations de l'emploi en France, coll. Repères, La découverte, Paris

Jolivet, A. (2003): Partir en retraite plus tôt ou plus tard : quelles implications d'une ouverture des marges de choix individuel ? in: La Revue de I'IRES 43, S. 37-57

Le Friant, M. (2006): Neue Arbeitsverträge: Mittel im Kampf gegen Arbeitslosigkeit?, in: Arbeit und Recht 12, S. 423

Lefèvre, G./Michon, F./Viprey, M. (2002): Les stratégies de entreprises de travail temporaires, Travail et Emploi 89, S. 45-64

Letremy, P./Meilland, C./Cottrell, M. (2004): Using working patterns as a basis for differentiating part-time employment, in: European Journal of Economic and Social Systems 17/1-2, S. 29-40

L'Horty Y. (2004): Instabilité de l'emploi : quelles ruptures de tendance? Les Papiers du CERC 1

Méda, D./Vennat, F. (sous la dir.) (2004): Le travail non qualifié, permanences et paradoxes, coll. Recherche, La Découverte, Paris Mückenberger, U. (2007): Ziehungsrechte - Ein zeitpolitischer Weg zur "Freiheit in der Arbeit", in: WSI-Mitteilungen 4, S. 195-201 OECD (2004): Employment Outlook, Paris

Palpacuer, F./Seignour, A./Vercher, C. (2007): Sorties de cadre(s), coll. Entreprise et société, La Découverte, Paris

Paugam, S. (2000): Le salarié de la précarité, PUF, Paris

Schmid, G./Gazier B. (ed) (2002): The Dynamics of Full Employment, Social Integration by Transitional Labour Markets, Edward Elgar, Cheltenham UK, Northampton, MA, USA

Supiot, A. (1994): Critique du droit du travail, PUF, Paris

Supiot, A. (1999): Au delà de l'emploi, Rapport pour la Commission européenne, Flammarion, Paris (Der Wandel im Arbeitsrecht und die Zukunft des Arbeitsrechts in Europa)

Tuchszirer, C. (2005): Le reclassement des salariés licenciés pour motif économique : responsabilité de l'entreprise ou de la collectivité publique ? La Revue de I'IRES 47, S. 157-173

Zachert, U. (2004): Beendigungstatbestände im internationalen Vergleich, Baden-Baden 